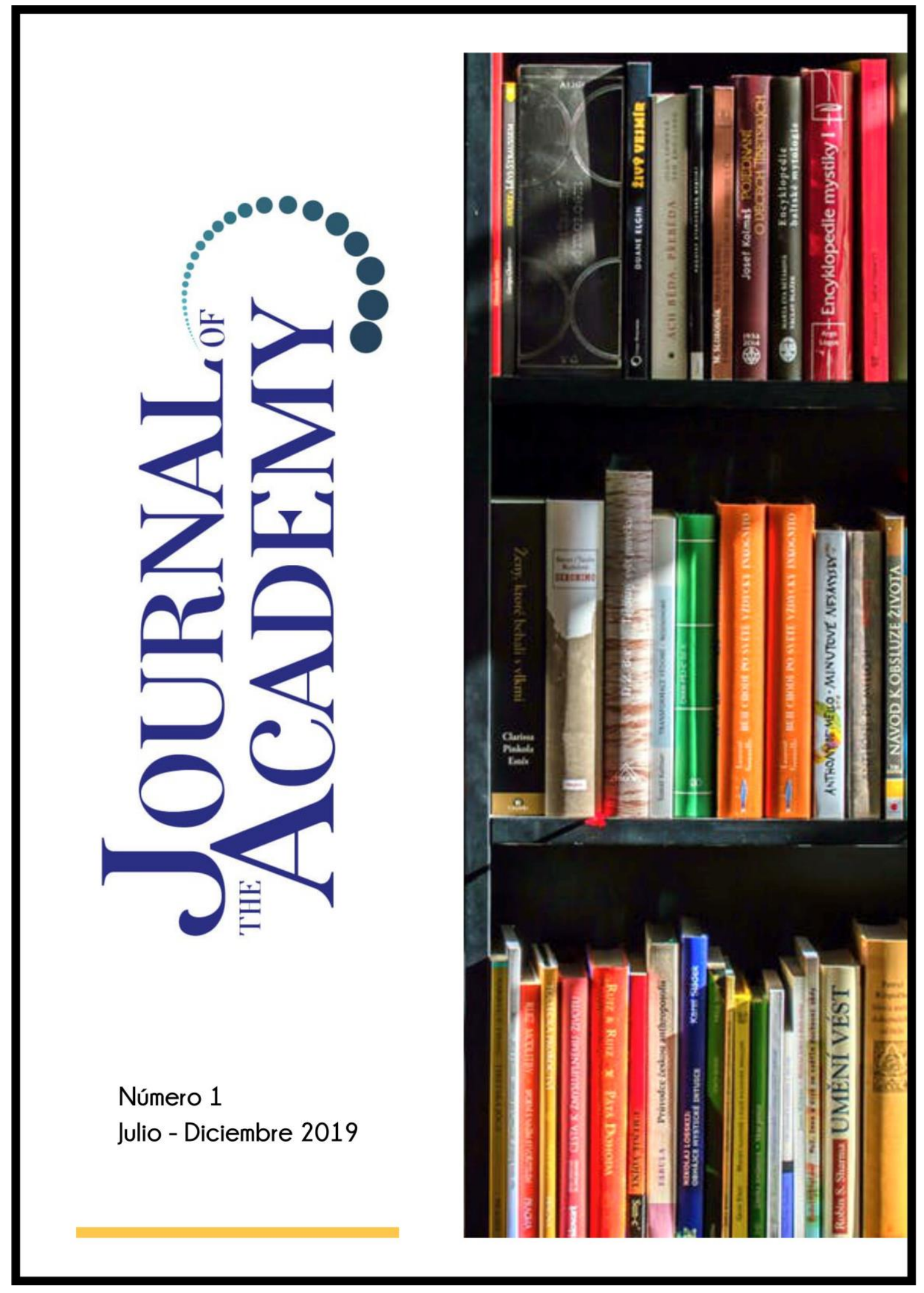




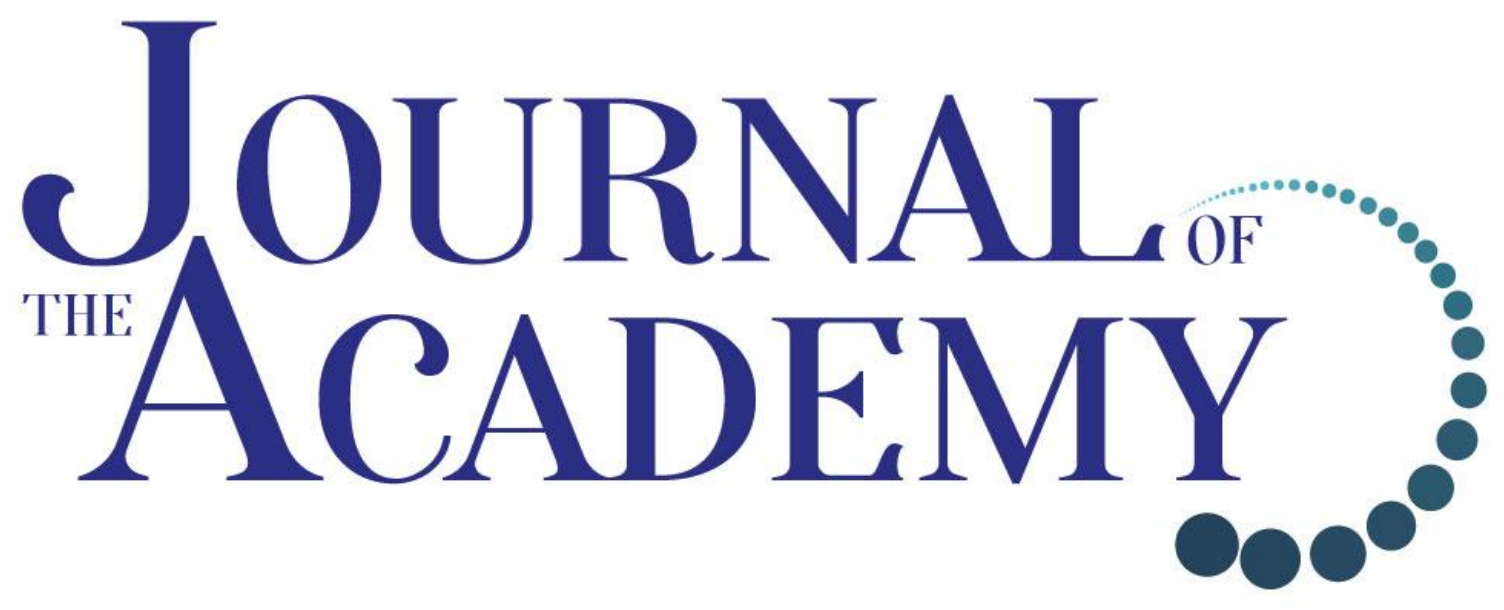

\section{Publicación Científica de la Asociación de Universidades del Perú ASUP}

Número 1

Julio-Diciembre 2019

www.journalacademy.org 
ISSN 2707-0301

\author{
Directora-Editora en Jefe \\ Ada Gallego Ruiz Conejo \\ Editor Científico \\ Oswaldo Orellana Manrique \\ Comité Científico \\ Javier Carreón Guillén \\ Universidad Nacional Autónoma de México, México \\ Martino Contu \\ Universidad de Sassari, Italia \\ Jorge Enrique Elías Caro \\ Universidad del Magdalena, Colombia \\ Roberto Escalante Semerena \\ Universidad Nacional Autónoma de México, México \\ Oscar Ortega Arango \\ Universidad Autónoma de Yucatán, México \\ Alex Veliz Burgos \\ Universidad de Los Lagos, Chile \\ Comité Editorial \\ Manuela Garau \\ Centro Studi Sea, Italia \\ José Manuel González Freire \\ Universidad de Colima, México \\ Carlos Tulio da Silva Medeiros \\ Diálogos en Mercosur, Brasil \\ Eduardo Gomes Onofre \\ Universidade Estadual da Paraíba, Brasil \\ Equipo Ejecutivo \\ Juan Carlos Norabuena Castañeda \\ Thalia Chávez Cortéz \\ Journal Academy \\ Revista Semestral Open Access
}




\title{
Reflexiones a 100 años del Manifiesto de Córdoba
}

\section{0-year reflections of the Cordoba Manifesto}

Recibido: 12 de julio de 2018

Aceptado: 23 de marzo de 2019

\author{
Oscar GARRIDO ÁLVAREZ \\ Universidad de Los Lagos, Chile \\ ogarrido@ulagos.cl \\ Omar ALTAMIRANO O. \\ Universidad de Los Lagos, Chile \\ oaltamirano@ulagos.cl
}

\begin{abstract}
The already 100-year old Liminal Manifest of the University Reform of Cordoba is still in the eyes of the intellectual and university world, reasons of the interest are named in several speeches and publications that allude to its importance and the defense of ideals that remains in the actual disputes of the students' movements. Thereon, its relevance and permanent analysis.
\end{abstract}

Keywords: Liminal Manifest, student movement, university reform

Resumen: El Manifiesto Liminar de la Reforma Universitaria de Córdoba, a 100 años de proclama concita aún el interés del mundo universitario e intelectual, las razones del interés están enunciadas en diversos discursos y publicaciones que aluden a su importancia y la reivindicación de consignas que no han dejado de estar en las actuales contiendas de movimientos estudiantiles. De ahí su relevancia y permanente análisis.

Palabras claves: Manifiesto liminar, movimiento estudiantil, reforma universitaria

\section{Para Citar este Artículo:}

Garrido Álvarez, Oscar y Altamirano O., Omar. Reflexiones a 100 años del Manifiesto de Córdoba. Journal of Academy num 1 (2019): 01-07.

Licencia Creative Commons Atributtion Nom-Comercial 3.0 Uunported

(CC BY-NC 3.0)

Licencia Internacional 


\section{Introducción}

La historia la construyen los hechos relevantes que han sucedido y que marcan una época, generando precedentes y consecuencias notables de estudiar y recordar. El manifiesto de Córdoba es una hecho que numerosos estudios lo consagran como un antes y un después en la historia latinoamericana de lucha de movimientos sociales, en particular el movimiento estudiantil, es por ello, que su aniversario nos invita a reflexionar en torno a sus demandas y la influencia que un grupo de entusiastas jóvenes influenciados por las ideas de cambio, en un contexto histórico particular, lograron permear la sociedad Argentina, e influenciar en movimientos universitarios y sociales en Latinoamérica.

¿Cuál es la vigencia de los planteamientos de la Reforma a 100 años? Es lo que nos aventuraremos a plasmar en las páginas siguientes, evidenciando su legado y los desafíos que en la actualidad pudiesen mantenerse o cobrar un nuevo sentido.

El artículo está organizado en primer lugar en un breve contexto histórico para situar el hecho y sus consecuencias: la configuración de la sociedad Argentina, los hechos en el mundo que tuvieron injerencia y las ideas que lo influenció. En segundo lugar, se analizarán las proclamas en sus aspectos más relevantes, sus principales postulados y arengas, evidenciando su coyuntura actual y, por último, se aventurará en analizar la real importancia del manifiesto de Córdoba y sus proyecciones futuras.

El documento no pretende ser un análisis exhaustivo desde sus distintas aristas, pero si relevar la connotación que en el mundo universitario esta disputa tuvo y tiene en la actualidad, y la relevancia en un contexto de políticas postneoliberales y de asociación internacional. La metodología de análisis es de carácter exploratorio de análisis de contenido de fuentes primarias y secundarias de autores contemporáneos que han analizado la reforma universitaria de córdoba

\section{Contexto histórico de la Reforma}

La reforma universitaria de 1918 está situada en un contexto histórico particular y hechos que la desencadenas, que si bien, a nivel local son particulares a la Argentina de principios de siglo XX, no son distintos a otros países latinoamericanos. A nivel global el contexto es el mismo, una guerra mundial que vivía su consumación y la Revolución Rusa que instala el primer gobierno socialista en el mundo. En Latinoamérica, la creación del primer partido comunista en Cuba y la revolución mexicana (Biagini, 2000: 108). Se suma a estos hechos, la influencia de los ideales socialistas y socialdemócratas de Europa que van generando un clima propicio para la concreción del manifiesto de Córdoba por un grupo de jóvenes idealistas que harían historia.

El contexto que enmarca el manifiesto en Argentina es bajo un gobierno radical asumido 1916 que por primera vez era elegido con voto universal, secreto y obligatorio. A nivel universitario la Ley Avellaneda de 1985 se convirtió en la primera ley que sancionaba y entregaba lineamientos para la organización administrativa y estructura jurídica de las universidades que en aquellos años eran solo dos: la Universidad de Buenos Aires y la Universidad Nacional de Córdoba. Esta última desconoció las reformas impuestas y mantuvo la organización tradicional con una importante influencia de la iglesia católica, con cuerpos directivos y académicos vitalicios sin importar su desempeño, donde las normas impuestas por la Universidad no permitían la disensión. Otro aspecto relevante es la formación de la sociedad argentina influenciada como ningún otro país latinoamericano por la inmigración europea y los ideales de libertad que importantes "revolucionarios" impregnaban en la sociedad. Como plantea Acevedo "Los acontecimientos de 
Córdoba fueron un triunfo de un movimiento estudiantil que supo aprovechar la coyuntura social de un país marcado por la permanencia de un sistema autoritario de viejas costumbres" (Acevedo, 2011: 1), agrega que el crecimiento de las clases medias inmigrantes y las inestabilidades políticas contribuyeron a crear un escenario ideal para las reformas, al igual las corrientes de pensamiento que se desarrollaban en el devenir de la primera guerra mundial, la Revolución Rusa y los movimientos sociales marcados por reivindicaciones obreras que acontecían alrededor del mundo. Tünnermann sitúa el movimiento de Córdoba como la primera confrontación entre la sociedad y una universidad en medio de cambios y esquemas obsoletos y en un parangón mayor plantea “...Universidad y sociedad marchaban sin contradecirse, pues durante los largos siglos coloniales y en la primera centuria de la República, la universidad no hizo sino responder a los intereses de las clases dominantes de la sociedad, dueñas del poder político y económico y, por lo mismo de la universidad" (Tünnerman, 2008: 37), por lo cual, para lograr comprender el movimiento de Córdoba es preciso situarse en su contexto socioeconómico y político.

Se alude también, a una confrontación de crisis de las elites tradicionales y el tránsito a una nueva generación intelectual que le importa lo social y lo colectivo sobre lo individual, que proclamaba transformaciones sociales y que repercutía a nivel continental, reconociendo a la reforma universitaria como un fenómeno político, genuinamente latinoamericano (Bruena, 2011:71-86). A principios de siglo XX se instalan en los países latinoamericanos ideales promulgados por distintos intelectuales, que reivindican el idealismo en oposición al positivismo que promulgaba Comte que había dominado al mundo, y sumado a anterior, la influencia de algunos pensadores latinoamericanos y de ellos en los jóvenes reformistas, entre los que se mencionan: El modernismo del poeta, periodista y diplomático nicaragüense Rubén Darío; el pensamiento emancipador y americanista del cubano José Martí; la crítica a la oligarquía y defensa de lo indígena del peruano Manuel González Prada; la crítica al racismo europeo y latinoamericano del mexicano José Vasconcelos, la defensa de lo americano y la crítica del utilitarismo norteamericano del uruguayo José Enrique Rodó; las ideas socialistas aplicadas a la realidad americana del argentino Manuel Ugarte y José Ingenieros (Pitelli, Hermo, 2010: 135-156). Todos ellos influenciaron de manera inequívoca en el Manifiesto de Córdoba que como veremos más adelante plasmo en sus consignas la crítica a la intrusión de otros países, y la valoración y defensa de lo latinoamericano.

En relación a los antecedentes descritos es necesario reflexionar sobre la reforma universitaria no como el resultado de una situación puntual, sino, del desarrollo de los acontecimientos que marcaron la época: a los hechos mundiales que acontecían, a las particularidades del país y situaciones propias de la Universidad de Córdoba, se venía incubando un naciente movimiento estudiantil que empezaba a organizarse no solo en Argentina, también en otros países de Latinoamérica. Los estudiantes de las principales universidades comenzaron a organizarse en centros de alumnos y a reunirse con sus pares de otras universidades, generando congresos y moldeando ideas comunes en encuentros internacionales de lo que debía ser la universidad latinoamericana. Se desarrollaron congresos internacionales en las ciudades de Uruguay en 1908, en Argentina y Colombia en 1910 y en Perú en 1912, se conformaron centros de estudiantes en distintos países, y en Argentina se creó la federación universitaria de la Universidad de Buenos Aires que fue desconocida por las autoridades, antecedentes que favorecieron un clima propicio al movimiento estudiantil (Biagini, 2000: 108). En la propia Universidad de Córdoba previo al Manifiesto Liminar se sucedieron una serie de hechos, como nuevas exigencias de asistencia de clases en Ingeniería y la supresión de internado para medicina que generaron descontento en los estudiantes, sumado a ello, la elección de un Rector que desconocen como legítima los alumnos 
organizados en la Federación Universitaria de Córdoba, gatillaron el estallido que algunos denominan como el "Grito de Córdoba" y el llamado a la juventud de Argentina y de Latinoamérica a revelarse a una sociedad que impedía los cambios y el acceso de la sociedad a nuevos conocimientos y corrientes de pensamiento.

\section{El Manifiesto Liminar}

El manifiesto liminar de 1918, fue publicado como un suplemento en el diario "La Gaceta Universitaria" creado el mismo año por los estudiantes universitarios. El manifiesto que convoca este artículo como el contexto histórico ya lo alude, ha tenido y tiene repercusiones que historiadores y analistas han resaltado en el trascurso de los años como un hecho histórico que funda una nueva universidad Latinoamérica y que marca el inicio de la lucha social que se proyecta más allá de solo una reivindicación de derechos de estudiantes universitarios. Para comprender el movimiento de los estudiantes de Córdoba se deben observar las dinámicas de poder de los grupos en conflicto, donde claramente se distinguen, el gobierno, la iglesia y la misma universidad (Palma, 2010:26) que yacían en anacrónicos principios que alejaban las nuevas formas de relacionarse con la sociedad, Palma también menciona a los trabajadores como grupo de conflicto, pero en este caso existía una mayor empatía en las reivindicaciones obreras.

El Manifiesto Liminar llama a dejar la "dominación monárquica y monástica" con un claro sentido nacionalista y secular, con una fuerte crítica a la injerencia de otros países que buscaban influir en el desarrollo de la sociedad latinoamericana y la presencia de la iglesia católica en la vida laica y su organización política y social. El Manifiesto es claro en manifestar "Estamos pisando sobre una revolución, estamos viviendo una hora americana" (Manifiesto Liminar, 2018), sin duda la lucha que se había gestado se había transformado en una revolución y así es reconocida por la historia, que sitúa el hecho como un punto de partida de muchas otras reivindicaciones que se originaron en el transcurso de las décadas posteriores.

El manifiesto plantea una fuerte crítica a las convenciones y prácticas de la Universidad de Córdoba que extrapola y caracteriza a demás universidades planteando que "Las universidades han sido hasta aquí el refugio secular de los mediocres, la renta de los ignorantes, la hospitalización segura de los inválidos y lo que es peor, el lugar donde todas las formas de tiranizar y de insensibilizar hallaron la cátedra que las dictara, las universidades han llegado a ser así fiel reflejo de estas sociedades decadentes que se empeñan en ofrecer el triste espectáculo de una inmovilidad senil. Por eso es que la ciencia, frente a estas casas mudas y cerradas, pasa silenciosa o entra mutilada y grotesca al servicio burocrático" (Manifiesto Liminar, 2018). La crítica es brutal a la estructura universitaria y a sus docentes que hasta ese momento gozaban de cargos vitalicios y no adecuaban sus conocimientos a nuevas corrientes de pensamiento y nuevas disciplinas de estudio. Se traza como la primera disputa entre una sociedad que comenzaba a experimentar cambios en su composición y una Universidad que se negaba a moverse de sus esquemas de antaño que solo privilegiaban a una clase social (Tünnermann, 2008: 163). La influencia del poder de la iglesia y de la clase oligárquica gobernante movió a los jóvenes universitarios a exigir democracia y una orientación social en la universidad, como señala Aboite "Estos jóvenes demandaban que la Iglesia y los poderes civiles sacaran las manos de la universidad, exigían democracia y pedían que la universidad tuviera una clara función social. Demandaban también libertad de expresión, libre acceso a las aulas universitarias y, en el fondo, planteaban que debía terminar la era de la universidad aristocrática y que esta debía abrirse de par en par a las nuevas clases sociales" (Aboites, 2008: 6). 
Los estudiantes de la federación universitaria de Córdoba se levantaron en contra de un régimen administrativo, contra un régimen docente, contra un concepto de autoridad y caracterizaban su lucha en que "Los métodos docentes estaban viciados en un estrecho dogmatismo, contribuyendo a mantener a la universidad apartada de la ciencia y de las disciplinas modernas. Las elecciones, encerradas en la repetición interminable de viejos textos, amparan el espíritu de rutina y de sumisión. Los cuerpos universitarios, celosos guardianes de los dogmas, trataban de mantener en clausura la juventud, creyendo que la conspiración del silencio puede ser ejercida en contra de la ciencia" (Manifiesto Liminar, 2018). Los postulados centrales del manifiesto giran en torno a la autonomía estudiantil, la libertad de cátedra y la educación laica, cuestionando la autoridad existente, la forma de enseñanza y las anacrónicas prácticas docentes. Varios son los análisis del manifiesto liminar, que destacan los principales postulados, entre ellos: la autonomía universitaria, la elección de los cuerpos directivos de las instituciones, la provisión de cargos de profesores por concurso, la libertad de cátedra, la gratuidad de la enseñanza, la reorganización académica, la asistencia social estudiantil, la vinculación con el sistema educativo nacional, el fortalecimiento de la función social de la Universidad a través de la extensión y la unidad latinoamericana (Tünnermann, 2008: 163; Samacá, Acevedo, 2011: 170-195; Acevedo, 2011: 137-155; Fernández, 2014: 663-687).

Si bien, todos los planteamientos son relevantes y la mayoría de ellos incuestionables hoy en día, el Ilamado a "la unidad latinoamericana" resulta ambicioso para la época, pero no menos relevante a la luz de la injerencia y proyección que alcanzó el movimiento a nivel latinoamericano y como algunos autores resaltan a nivel continental (Donoso, Contreras, 2017: 42-65). El manifiesto proclama el nacimiento de una verdadera revolución y llama a todos los hombres libres de América a alzar la voz y a luchar por la suprema libertad, es así que, otros movimientos universitarios y políticos en el continente americano adoptaron los principios de la reforma, lo que resalta la vanguardia de las ideas y pensamientos de los estudiantes (Pitelli, Hermo, 2010: 135156). El ideario reformista adoptó distintas figuras ideológica que según los países las reapropiaron y resignificaron siendo la génesis de partidos y movimientos políticos, la reforma también se enmarca en una crisis de civilización que proclamaba ideales humanistas y una renovación social (Suasnábar, 2018: 174-189). Otro aspecto importante de resaltar de la reforma es el sentido social o misión social al que apelaron los estudiantes, reconociendo que esta inquietud nace de los estudiantes como una demanda certera a la universidad y que hoy se reconoce como la apertura de la universidad latinoamericana a la extensión. Una nueva sensibilidad de los jóvenes para involucrarse en la sociedad, a percibir que su desarrollo debía ser en conjunto con la sociedad y que el conocimiento debía favorecer a la solución de las problemáticas sociales (Donoso, Contreras, 2017: 42-65). El nuevo cometido que adopta la universidad latinoamericana y proclamado por los estudiantiles que visualizaron a la universidad vinculada más estrechamente con la sociedad y sus problemas, se reconoce como resultado de la reforma, la incorporación de la extensión universitaria y la difusión cultural como una actividad normal de la universidad Latinoamérica, pero también, se alude a que este sentido social, no tiene el mismo alcance en las distintas universidades en la actualidad (Tünnermann, 2008:163).

\section{El legado}

El manifiesto de Córdoba en una mirada retrospectiva para analizar sus consecuencias y sus proyecciones futuras, resulta un análisis ambicioso en la actual diversidad de instituciones. Ya en el análisis precedente se han esbozado algunas ideas y las más desafiantes resultan ser la 
alocución a la transformación social y la renovación ideológica consistente con las ideas de la época, el llamado y desafío a la identidad común latinoamericana, la trasformación del estudiante en un sujeto activo en la dinámica universitaria, demandante frente a la institucionalidad y responsable de involucrarse con la sociedad, y quizás lo más relevante, es la redefinición del rol de la universidad formadora de elites políticas e intelectuales, a una universidad, que sin excluir lo anterior, formadora de profesionales, más inclusiva y masificada.

La universidad desde sus inicios que se remontan al siglo IX o XI según la mirada que demos a la historia, siempre ha mantenido una constante disputa por su autonomía en contra de la intrusión de poderes políticos, religiosos o económicos que pudiesen llevarla a defender intereses de grupos particulares. La universidad previa al manifiesto respondía a una lógica oligárquica liberal que se abocaba a formar las reducidas élites políticas y de intelectuales que definían el rumbo de la sociedad, frente a ello, esta larga disputa enfrenta a la anquilosada universidad con nueva universidad latinoamericana cimentada en el Manifiesto de Córdoba (Suasnábar, 2018: 174-189). La naciente universidad es una mixtura entre el modelo anglosajón de autogobierno y del modelo napoleónico que incorpora las ideas de igualdad de acceso, gratuidad y financiamiento estatal, todos conceptos proclamados por los estudiantes y reconociéndose en este hecho, el carácter fundacional de lo que forma parte de la nueva universidad latinoamericana (Pitelli, Hermo, 2010: 135-156). Tünnermann identifica tres características de la configuración de la universidad latinoamericana a partir del manifiesto de Córdoba: 1) la organización y gobierno de la universidad, con la conquista de la autonomía de otros poderes sociales, el cogobierno con la participación estudiantil en la elección de autoridades universitarias y la participación en la definición de las políticas institucionales por parte de los estudiantes; 2) la enseñanza y los métodos docentes, con la reorganización académica, los concursos para seleccionar a los profesores, la libertad académica para el análisis y expresión de ideas, entre otros; 3 ) la proyección política y social de la Universidad, delineando la intervención de la universidad en los temas de interés social e involucrándose con la sociedad en materias de extensión y cultura, y agrega, 4) la vocación americanista, con la postura antiimperialista y contraria a cualquier forma autoritaria de gobierno.

Los postulados de la reforma fueron muy esperanzadores para la época y más aun considerando la conservadora sociedad que se resistía a asumir las corrientes de cambios. También, se debe asumir que no todos los postulados se implementaron según los ideales reformistas y hoy en día siguen siendo parte de las luchas de movimientos estudiantiles.

La imposición de medidas neoliberales en las universidades ha causado deterioro en la autonomía, en la burocracia de las instituciones, en la exclusión de estudiantes y trabajadores de las decisiones, una universidad de gerentes centrados en entregar un servicio de calidad y responder a las directrices estatales (Aboites: 2008: 6-16). Se plantea que si en el transcurso de estos 100 años, se hubiesen desarrolla plenamente los planteamientos de la reforma, la Universidad en América Latina sería muy diferente a la existente, y se reconoce además, la influencia negativa de las dictaduras militares en Latinoamérica en el pasado siglo con la implementación de políticas neoliberales y el abandono de políticas nacionales por parte del Estado, en la dispar implementación de las demandas de los estudiantes de Córdoba (Fernández, 2014: 663-687). El mismo autor lista una serie de características de la universidad latinoamericana contemporánea:

- Fuerte diversificación, segmentación y heterogeneidad de los niveles de calidad.

- Gran crecimiento del número de universidades privadas, muchas de ellas de muy bajo nivel académico. 
- Crecimiento inorgánico del número de universidades y de estudiantes, cursando carreras no vinculadas con las necesidades actuales y futuras de los países.

- Escasa articulación entre la educación superior universitaria y no universitaria- con el resto del sistema educativo, particularmente la enseñanza media.

- Falta de flexibilidad, innovación y actualización científica de los diseños curriculares y de los programas de las asignaturas.

- Escasa articulación entre las instituciones de educación superior con la sociedad, el trabajo y la producción, y poca participación social en el desarrollo universitario.

- Rigidez en los modelos organizativos y en las estructuras de gestión académica, afectando la necesaria flexibilidad para la planificación y el desarrollo universitario.

- Políticas nacionales e institucionales de desarrollo con lineamientos excesivamente genéricos, sin atender adecuadamente las perspectivas de mediano y largo plazo y sin proyectos específicos para hacerlos efectivos.

- Escaso gasto público en investigación para el desarrollo (I+D).

Las características planteadas no son ajenas a los análisis de la educación superior que se realizan en la actualidad y la mayoría de ellas ni siquiera podrían haber sido demandas posibles hace 100 años atrás, tampoco el Manifiesto de Córdoba pretendía avanzar en la implementación de sus proclamas sin oposición, fue el inicio de un movimiento que permitió abrir la universidad a los alumnos excluidos por su origen o posición social, permitió el acceso a la academia a nuevos profesionales e intelectuales, permitió la participación estudiantil en el gobierno universitario y permitió vincular a la universidad con las necesidades reales de la sociedad. Esas son las consignas que cobraron mayor valor y aun así en la actualidad se presentan matices de las mismas, pero que no se discuten como características propias de la universidad latinoamericana. También es cierto que la permanencia de las ideas reformistas planteadas en el año 1918 radica en la ductilidad de las ideas que le dieron origen y de ahí su vigencia y permanente invocación (Suasnábar, 2009: 5161).

\section{Conclusiones}

La vigencia de los planteamientos del movimiento estudiantil radica en la reforma del gobierno universitario y la reforma docente con todos los alcances que ello tuvo, también, en el nuevo enfoque social que se expresa en la preocupación de la nueva universidad Latinoamérica, que deja el encierro que la identificaba para evolucionar en un actor relevante en la transformación social. En sus inicios el enfoque social se expresó más nítidamente en el ingreso de jóvenes hasta ese entonces excluidos de la formación universitaria y en la extensión universitaria y cultural abierta a la ciudadanía, conocida en la actualidad como responsabilidad social universitaria. Como se desprende de los argumentos planteados, la reforma no solo fueron aspectos académicos, su mirada fue más allá de las aulas, también tuvieron alcances políticos y sociales, proclamando ideas nacionalistas, el populismo, el laicismo y la democracia, y contrarios a ideas imperialistas y totalitarismos.

A 100 años de la reforma es necesario volcar la mirada a un escenario distinto de los países de Latinoamérica, donde los slogan, si bien no han cambiado, se incorporan otras luchas asociadas a otras conquistas relevantes de validar para las universidades, entre ellas se encuentran: los temas de género, los inmigrantes, los pueblos originarios, los discapacitados y la apertura a grupos no considerados, como los jóvenes de familias más pobres, que ven en una carrera universitaria el anhelo de la movilidad social. La inclusión social que busca que todas las personas tengan las 
mismas oportunidades para participar en la sociedad, se ha vuelto para las universidades en una condición relevante en su quehacer, sobretodo, cuando el acceso a la educación superior producto de las mayores oportunidades a jóvenes de estratos socioeconómicos más bajos, se ha acrecentado en países latinoamericanos. En este caso el desafío es mayor, cuando la diversificación de instituciones privadas producto de políticas neoliberales genera serias críticas a la calidad de las instituciones y de los alumnos que forman.

La sociedad moderna también implica desafíos importantes a la universidad Latinoamérica, la sociedad del conocimiento y las nuevas tecnologías han transformado a la sociedad, el acceso a la información que puede caracterizarse de ilimitado e inmediato estampa a las universidades una nueva forma de plantearse frente al conocimiento, impulsando la investigación científica y generando las condiciones que permitan un mejor desarrollo de la innovación. Los desafíos también involucran una gestión más eficaz, frente a limitaciones presupuestarias, rigideces de criterios de autoevaluación de agencias externas o exigencias especiales de normativas gubernamentales, a las que las universidades deben responder y afrontar, sin que ello implique dilaciones a sus funciones inherentes.

Hoy, desde el ideario reformista se hace más latente la colaboración de las instituciones de educación superior de los países latinoamericanos, para asumir los nuevos desafíos que enfrenta la universidad, una integración entendida desde la contribución al desarrollo de los países, con una mirada de corto y largo plazo. La colaboración y asociación entre universidades latinoamericanas para establecer redes académicas que estimulen el intercambio de estudiantes y docentes, y permitan generar colaboración en las áreas propias de la universidad en docencia, investigación y extensión.

\section{Referencias bibliográficas}

Aboites, H. (2008), El futuro de la universidad en América Latina, Revista Acción Educativa, 8, pp. 6-16.

Acevedo, A. (2011), A cien años de la reforma de Córdoba 1918-2018, la época, los acontecimientos, el legado, en Revista Historia y espacio 7(36), pp. 137-155.

Biagini, H. (2000), La Reforma Universitaria, Antecedentes y Consecuencias. Buenos Aires: Editorial Leviatán.

Bruena, L. (2011), La Reforma Universitaria como fenómeno político y su proyección latinoamericana, en Revista Cuadernos del Ciesal, 9, 73-86.

Donoso, A. y Contreras, R. (2017), La dimensión social del movimiento estudiantil de Córdoba den 1918, Revista Izquierdas 33, pp. 42-65.

Fernández, N. (2014), Universidad, sociedad y conocimiento, reflexiones para el debate, Revista Avaliação de educación superior, 9(3), pp. 663-687.

Palma, C. (2010), Reforma de Córdoba: grito de resistencia y quiebre oligárquico, Revista Izquierdas, año 3(8) 1-26. 
Pittelli, C. y Hermo, J. (2010), La reforma universitaria de Córdoba (Argentina) de 1918. Su influencia en el origen de un renovado pensamiento emancipatorio en América Latina, Revista Online Historia de la Educación, 29, pp. 174-189.

Samacá, G. y Acevedo, Á. (2011), De la reforma de Córdoba al Cordobazo. La universidad como escenario de las luchas por la democracia en Argentina, 1918-1969 y su impacto en Colombia, Revista Memorias. Revista digital de Historia y Arqueología desde el Caribe, 8(15), pp. 170-195.

Suasnábar, C. (2009), La reforma universitaria de Córdoba de 1918: una mirada histórica de la relación entre intelectuales, universidad y política en la Argentina, Revista Práxis Educativa, 4(1) pp. 51-61.

Suasnabar, C. (2018), Legado, crítica y superación del ideario de la reforma universitaria de 1918: Julio V. González y la radicalización del pensamiento reformista, Revista História da Educação, 22(54), pp. 135-156.

Tünnermann, C. (2008), 90 años de la reforma universitaria (1918-2008). Nicaragua: Editorial Hispamer. 